\title{
CARACTERIZACIÓN HIDROGEOLÓGICA DE LA CUENCA DEL RÍO LAS MARÍAS, LOS CÓBANOS, SONSONATE, EL SALVADOR
}

\author{
HYDROGEOLOGICAL CHARACTERIZATION OF THE RIVER BASIN LAS \\ MARÍAS, LOS COBANOS, SONSONATE, EL SALVADOR
}

\author{
Celia E. Monge ${ }^{1 *}$, Ingrid Vargas ${ }^{2} \&$ Dagoberto Arévalo ${ }^{1,3}$ \\ ${ }^{1}$ Administración Nacional de Acueductos y Alcantarillados, El Salvador \\ ${ }^{2}$ Escuela Centroamericana de Geología, Universidad de Costa Rica, Costa Rica \\ ${ }^{3}$ Universidad de El Salvador, El Salvador \\ *Autora para contacto: celia_monge@hotmail.com
}

(Recibido: 04/07/2015; aceptado: 15/02/2016)

\begin{abstract}
Hydrogeological characterization of the river basin Las Marias, Los Cóbanos department of Sonsonate, El Salvador, was performed with the aim of evaluating the aquifer system. The analysis included the use of hydrogeochemical and geophysical tools, and lithological information available in the study area, which allowed us to propose a conceptual model of the aquifer and exclude the presence of seawater intrusion. The existence of two aquifers was determined in the area, one of them is a shallow aquifer, mid production with a variable thickness of 0 meters to 20 meters, and is used in the area for water supply through dug wells with depths not exceeding 6 meters and diameters up to 1.5 meters. Analysis of the chemical data determined that there are anthropogenic pollution sources in the area that affect the water quality. Another aquifer was detected with the vertical electrical tests and which are known to exist by private drilled wells supplying hotels, farms and private properties in the area. A water balance showed that the 1739 $\mathrm{mm}$ annual rainfall, $70.6 \%$ equivalent to $1217 \mathrm{~mm}$ corresponds to actual evapotranspiration, $13 \%$ (226 $\mathrm{mm}$ ) belongs to the retention by vegetation and the remaining $16.4 \%(278 \mathrm{~mm})$ represents the water recharge zone.

Keywords: Hydrogeological conceptual model, seawater intrusion, hydrogeochemical, river basin Las Marías, El Salvador.

RESUMEN: Se realizó una caracterización hidrogeológica de la cuenca del río Las Marías, Los Cóbanos, departamento de Sonsonate, El Salvador, con el objetivo de evaluar el sistema acuífero. El análisis incluyó el uso de herramientas hidrogeoquímicas, geofísicas y la información litológica disponible en la zona de estudio, la cual permitió plantear un modelo conceptual del acuífero y descartar la presencia de intrusión salina. Se determinó la existencia de dos acuíferos en la zona, el primero de ellos es un acuífero somero, de mediana producción, con un espesor variable de $0 \mathrm{~m}$ hasta 20 $\mathrm{m}$, y es el que la población de la zona utiliza como fuente de abastecimiento de agua a través de pozos excavados con profundidades que no superan los $6 \mathrm{~m}$ y diámetros de hasta $1,5 \mathrm{~m}$. De los análisis químicos se identificó la influencia de fuentes de contaminación antropogénica en la calidad del agua. Un segundo acuífero fue identificado con los sondeos
\end{abstract}

MONGE, C. E., VARGAS, I. \& ARÉVALO, D., 2016: Caracterización hidrogeológica de la cuenca del río Las Marías, Los Cóbanos, Sonsonate, El Salvador.- Rev. Geol. Amér. Central, 55: 185-195, DOI: 10.15517/rgac.v55i0.27074 
eléctricos verticales y posiblemente esté siendo captado por pozos perforados privados que se ubican fuera del área de investigación. Mediante la elaboración de un balance hídrico se determinó que de los $1739 \mathrm{~mm}$ de precipitación anual, el 70,6\% equivalente a $1217 \mathrm{~mm}$ corresponde a evapotranspiración real, el 13\% (226mm) pertenece a la retención por la vegetación y el restante $16,4 \%$ (278mm) representa la recarga acuífera de la zona.

Palabras clave: Modelo hidrogeológico conceptual, geofísica, hidrogeoquímica, Cuenca Las Marías, El Salvador.

\section{INTRODUCCIÓN}

El área de estudio se localiza en la cuenca del río Las Marías, entre las coordenadas Lambert 266000-280000 Norte y 410000-417000 Este, está situada en la región occidental de la República de El Salvador, aproximadamente a 11 $\mathrm{km}$. al suroeste de la ciudad de Sonsonate, pertenece a los municipios de Acajutla y Sonsonate, ambos del departamento de Sonsonate. En la figura 1 se muestra el detalle de ubicación.

La cuenca del río La Marías comprende un área de $19 \mathrm{~km}^{2}$, la elevación máxima de la zona de estudio es de 100 m.s.n.m. y desemboca al mar en la playa de Los Cóbanos situada al este del Puerto de Acajutla.

El uso de la tierra en la cuenca del río Las Marías es muy diverso; en la parte alta de la cuenca existen cultivos de caña de azúcar, y parcelaciones habitacionales que no cuentan con servicio de agua potable ni alcantarillado sanitario, la porción media de la cuenca está dedicada principalmente a los cultivos de maíz, sorgo y frijol, intercalados con algunos establos de crianza de ganado y parcelas habitacionales; la parte inferior de la cuenca cuenta con parcelaciones habitacionales sin servicio de agua potable ni alcantarillado sanitario en contraposición a una gran infraestructura turística representada por hoteles y restaurantes. La desembocadura del río La Marías alberga una gran diversidad ecológica que está conectado a las únicas formaciones coralinas que se conocen en la Costa del Océano Pacífico por lo que este lugar ha sido declarado como Área Natural Protegida (MARN, 2007).

El interés por el estudio de esta zona se basa principalmente en establecer las características del acuífero somero existente y debido a que este es la fuente de abastecimiento de la población rural, es necesario determinar las condiciones de la calidad del agua del acuífero superior e identificar una posible cuña de intrusión marina.

\section{RASGOS GEOLÓGICOS E HIDROGEOLÓGICOS}

Geomorfológicamente, el área de investigación está situada dentro de la llamada Planicie Costera de Sonsonate, la planicie principalmente está formada por rocas volcánicas del Cuaternario provenientes de los centros volcánicos ubicados al norte del área, también existen en la zona lahares y depósitos aluviales compuestos de gravas, arenas, limos y arcillas que han sido transportados por las corrientes de agua de las partes altas a las bajas donde han sido depositados (PNUD, 1981). En menor medida se observan depósitos volcánicos del Terciario, esencialmente lavas andesíticas y basálticas (PNUD, 1981)

La región presenta características morfológicas propias de los fenómenos tectónicos volcánicos como levantamientos, erupciones volcánicas y hundimientos que han sucedido a través de diferentes tiempos geológicos (PNUD, 1981), esto puede observarse en toda la región y principalmente a lo largo de la carretera del litoral.

En la zona existe un sistema de fallas tectónicas transversales que ha dado origen a una amplia extensión de hundimiento y separación de la continuidad de la unidad geomorfológica denominada Cadena Costera. (Estévez, 2007).

Regionalmente, campañas geológicas precedentes han distinguido la presencia de dos formaciones geológicas predominantes: Bálsamo y San Salvador. El término Estratos de Bálsamo 


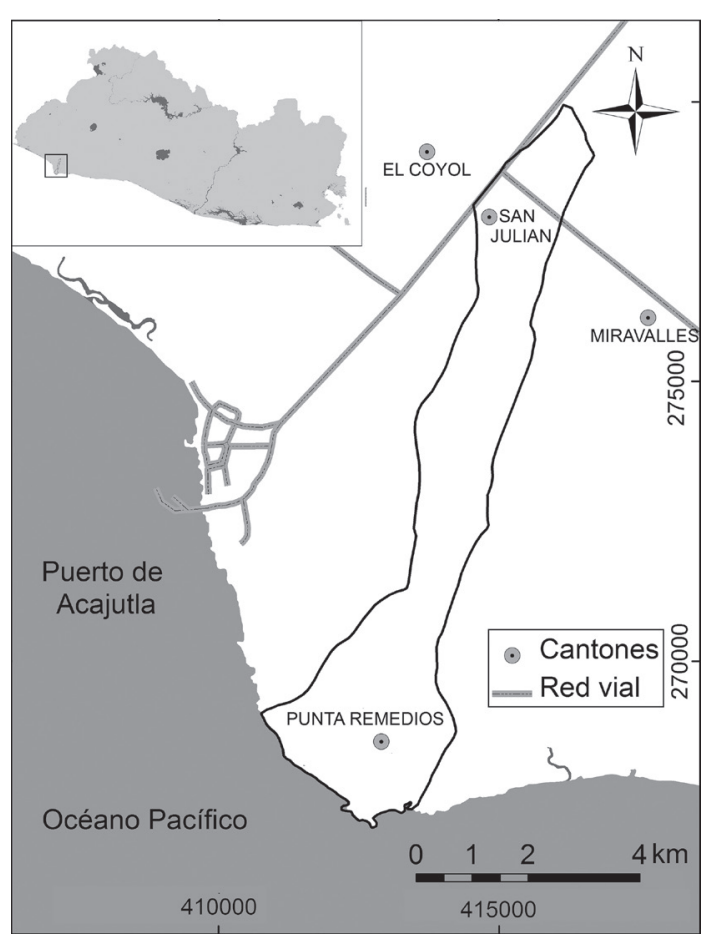

Fig. 1: Mapa de ubicación de la cuenca del río Las marías.

fue introducido por Dürr (1960) y posteriormente Wieseman (1975) lo define como una Formación geológica del mismo nombre y lo subdivide en tres miembros geológicos: miembro b1, b2 y b3 (Baxter, 1984). Consistentes en rocas que van desde ácidas, intermedias y básicas.

El término Estratos de San Salvador, fue introducido por Dürr (1960) y los divide en serie Superior e Inferior por la presencia de suelos fósiles, posteriormente Wieseman (1975) lo define como una formación geológica (Baxter, 1984). La secuencia consiste en piroclastitas ácidas y efusivas ácidas-básicas intercaladas (Baxter, 1984).

La verificación de la geología local de la cuenca del río Las Marías se desarrolló mediante inspecciones de campo, a partir de las cuales se identificaron rocas volcánicas asociadas con dos formaciones: las brechas y lavas andesíticas que se encuentran en forma de cerros relictos y al sur de la cuenca del río Las Marías corresponden a la Formación Bálsamo, y las tobas líticas con pómez que cubren la mayor parte de la cuenca asociadas con la Formación San Salvador.
Formación Bálsamo: en la zona de estudio se identificaron depósitos masivos, brechosos principalmente hacia el sur del río Las Marías, estos consisten de brechas soldadas con fragmentos angulares de lava, color gris cuando está sana y rojiza cuando se encuentra meteorizada, los fragmentos son centimétricos y presentan una textura porfirítica y composición andesítica. Se observaron cortes con aproximadamente diez metros de espesor. Aunque la morfología del terreno es típicamente plana, existen pequeñas colinas (con elevaciones variables que pudieran alcanzar hasta los $15 \mathrm{~m}$ ) compuestas de bloques métricos, subangulares de lava de composición andesítica basáltica, en las márgenes del río y otras sobreyaciendo los depósitos brechosos. Todos estos depósitos se asocian al Miembro b1 de la Formación Bálsamo que comprende del periodo Plioceno al Mioceno (Baxter, 1984).

Formación San Salvador: se ha descrito como piroclastitas ácidas y epiclastitas volcánicas, efusivas básicas (Machuca, 2003). En la zona de estudio se encontraron afloramientos de tobas líticas con clastos de pómez principalmente en el margen del río Las Marías y en los pozos artesanales excavados por la población de la zona. Aproximadamente un $75 \%$ del área de estudio está cubierta con estos materiales. Los depósitos en el margen del río Las Marías consisten en depósitos soldados meteorizadas heterogéneas, compuestas por líticos centimétricos de color beige predominantemente, que además contienen fragmentos de pómez meteorizada. En otros afloramientos masivos, subhorizontales, se observan tobas retrabajadas con fragmentos líticos grises y rojizos, y otros tabulares vítreos. En dichos afloramientos se determinaron espesores visibles de hasta $5 \mathrm{~m}$.

Hacia el sur de la cuenca Las Marías, en la sección de pleamar, se distingue una formación de rocas sedimentarias que sobreyacen a las epiclastitas del miembro b1 de la Formación Bálsamo. Este afloramiento de areniscas, color café, de grano medio, con fragmentos de conchas y elementos calcáreos ricos en carbonatos que actúan como el cementante, se han identificado con el nombre de Coquinas Los Cóbanos. Estas rocas sedimentarias únicamente se han identificado en esta área 
en El Salvador, por lo que su origen se asocia a los esqueletos calcáreos de los arrecifes de coral existentes en la esta zona.

El Mapa Hidrogeológico de El Salvador publicado en 2008 por ANDA-COSUDE resalta dos unidades hidrogeológicas en la zona de estudio tal como se observa en la figura 2, la Unidad Acuífero Poroso de Gran Extensión y Productividad Media, caracterizada por rocas piroclásticas, aglomerados y retrabajados (pómez, lapilli, tobas), con una distribución granulométrica que varía de fina a gruesa. La dirección del flujo del agua subterránea en esta unidad varía del SW al SE, mientras que las conductividades hidráulicas pueden variar de medianas a bajas, como consecuencia al grado de cementación o compactación que pueden tener los granos de los materiales que la constituye. Esta unidad puede tener más de $50 \mathrm{~m}$ de espesor. La Unidad de Rocas No Acuíferas, está constituida por flujos macizos de lavas, intercalados con tobas aglomeradas y brechosas, además de lahares cementados, tienen conductividades hidráulicas muy bajas o casi nulas, debido a su baja porosidad o a su alto grado de compactación y cementación. Se considera que constituyen el basamento de los acuíferos identificados en la zona de estudio. La profundidad a la que se encuentra esta unidad puede superar los $100 \mathrm{~m}$.

\section{RESULTADOS}

\section{Métodos geoeléctricos}

El método geofísico utilizado fue el de resistividad eléctrica, el cual es un procedimiento geoeléctrico que induce corrientes eléctricas artificiales en el terreno y las diferencias de potencial resultantes se miden en la superficie. Dicho método se utiliza en el estudio de las discontinuidades horizontales y verticales de las propiedades eléctricas del suelo, así como en la investigación de cuerpos tridimensionales con anomalías en la conductividad eléctrica. (Kearey, Brooks \& Hill, 2002).

Para la aplicación de sondeos eléctricos verticales se empleó un equipo de resistividad McOHM 2115A, con el que se utilizaron dos

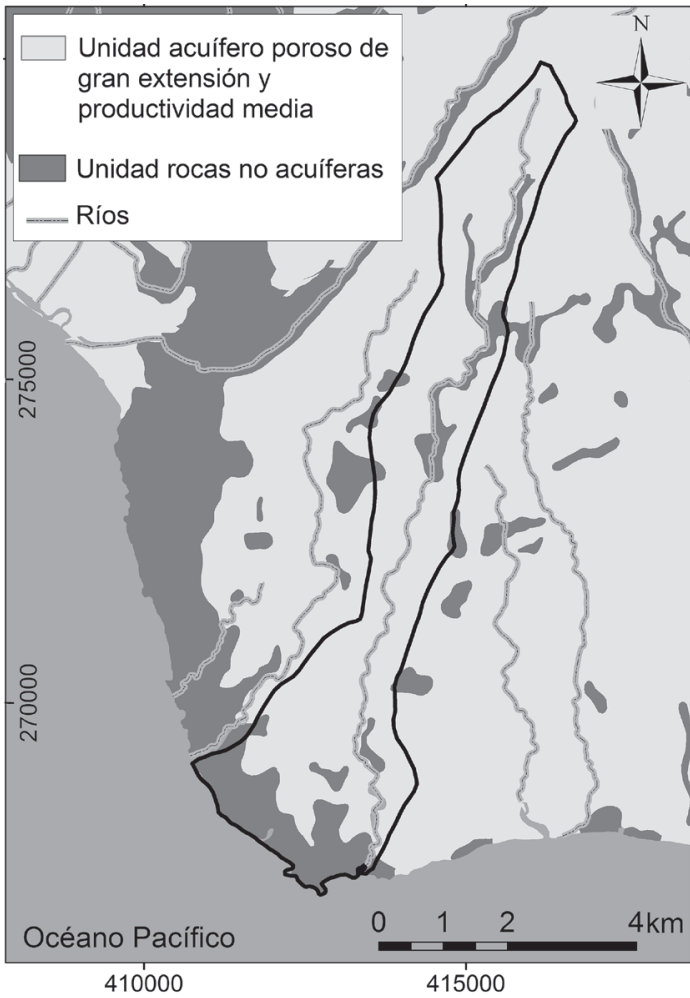

Fig. 2: Mapa hidrogeológico en la zona de estudio. (Fuente: ANDA-COSUDE, 2008).

electrodos de corriente $\mathrm{AB}$ y dos electrodos de potencial $\mathrm{MN}$, este equipo se utilizó para el arreglo Schlumberger, el cual es un dispositivo de configuración simétrica, donde la distancia entre los electrodos detectores MN es mucho menor que la distancia de los electrodos inyectores $\mathrm{AB}$. Los electrodos $\mathrm{MN}$ se mantienen fijos respecto a un punto central mientras que los electrodos $\mathrm{AB}$ se separan progresivamente para aumentar la profundidad de investigación.

Se realizaron 12 sondeos eléctricos verticales dentro del área de estudio con la colaboración del personal y equipo de Hidrogeología de la Administración Nacional de Acueductos y Alcantarillados ANDA (2010), los cuales se interpretaron con el programa IPI2win y con ellos se elaboraron 5 perfiles geoeléctricos, además se cuenta con información de 15 sondeos eléctricos realizados por GEOCEL (1992) en la zona de estudio, los cuales se reinterpretaron y de ellos se 
elaboraron 3 perfiles geoeléctricos. La ubicación de todos los sondeos se muestra en la figura 3. A partir de los 8 perfiles geoeléctricos, en conjunto con la interpretación de las características litológicas de un pozo en la zona de Acajutla, más la geología de campo y los niveles estáticos de los pozos excavados, se definieron las diferentes capas del subsuelo y sus respectivas resistividades para cada uno de los 8 perfiles geoeléctricos.

Dado que todos los perfiles son iguales, se muestra como ejemplo el perfil A - A' en la figura 4 donde se observa la existencia de una primera capa de resistividad de $2,4 \Omega \mathrm{m}$ cuyo espesor es de hasta $18 \mathrm{~m}$, que corresponde con los depósitos aluviales con intercalaciones de piroclastos según lo observado en el campo. En esta capa se encuentra el nivel freático del acuífero somero en el que se ubican todos los pozos excavados de los que se abastecen de agua los pobladores de la cuenca del río Las Marías. La siguiente capa posee una resistividad aparente de $36 \Omega \mathrm{m}$ y espesor aproximado de $40 \mathrm{~m}$, la cual puede asociarse a la capa de tobas no saturadas. Luego se tiene una capa con una resistividad aparente de $7,6 \Omega \mathrm{m}$, de acuerdo a los SEV se encuentra a una profundidad de 60 $\mathrm{m}$, se considera una capa muy conductiva por lo que se puede interpretar como la zona en la que se encuentra un segundo acuífero, lo cual concuerda con sedimentos aluviales y piroclastos de acuerdo a la columna litológica del pozo en Acajutla.

Los resultados del estudio geofísico realizado por GEOCEL (1992) en el cual se utilizó una apertura de $\mathrm{AB} / 2$ de $500 \mathrm{~m}$, indica que teóricamente se logró investigar hasta profundidades de $150 \mathrm{~m}$ a $200 \mathrm{~m}$, y se encontró una capa por debajo de los $120 \mathrm{~m}$ con una resistividad entre los 4 a 9 , que en aquel momento los investigadores interpretaron como una zona de intrusión salina dado que no contaban con registros de perforación en la zona hasta esa profundidad.

La profundidad de penetración teórica mediante prospecciones geoeléctricas alcanzada para esta investigación fue de $100 \mathrm{~m}$. Los resultados de los SEV realizados a pocos metros de distancia del límite de costa, no reflejan que se haya interceptado alguna cuña de intrusión salina para el acuífero somero.

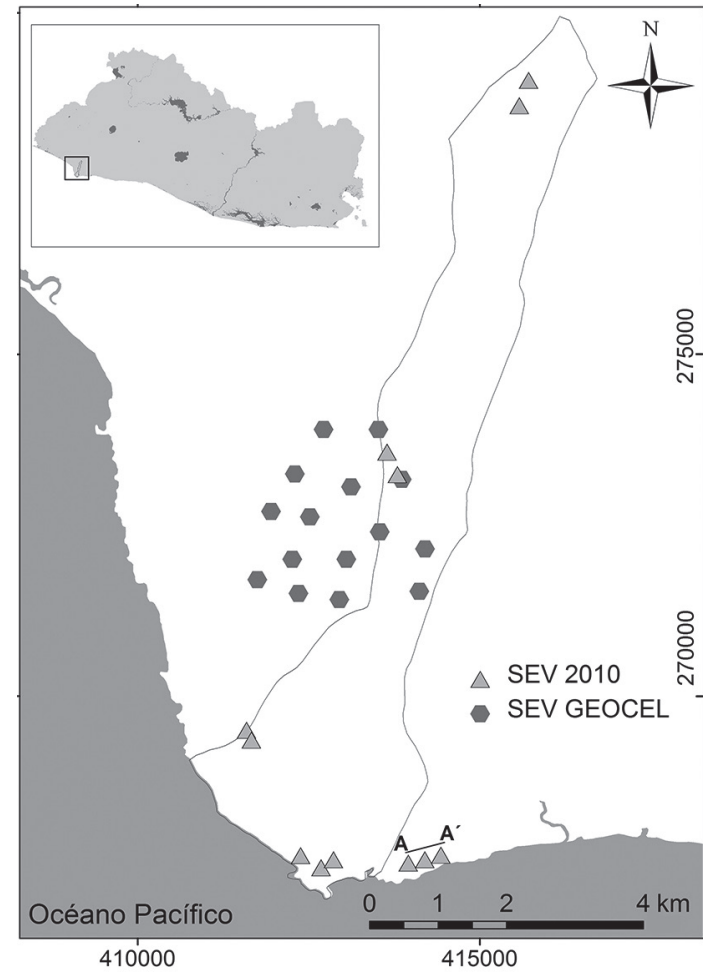

Fig. 3: Ubicación de sondeos eléctricos verticales y perfil longitudinal.

El balance hídrico realizado con la metodología de Schosinsky (2006) dio como resultado un valor medio de recarga potencial de $276 \mathrm{~mm} / \mathrm{año}$ equivalente a $174 \mathrm{l} / \mathrm{s}$. Conforme a los resultados del balance se obtuvo que de los $1739 \mathrm{~mm}$ de precipitación anual, el 70,6\% equivalente a $1217 \mathrm{~mm}$ corresponde a evapotranspiración real, el 13\% (226 $\mathrm{mm})$ pertenece a la retención por la vegetación y el restante $16,4 \%(276 \mathrm{~mm})$ representa la recarga acuífera de la zona. Como se puede notar no se tiene un valor de escurrimiento dado que la zona es muy plana y cuando el suelo alcanza la saturación la zona se encuentra anegada lo que mantiene aún las condiciones de humedal y su biodiversidad.

La transmisividad del acuífero superficial fue determinada a partir de la información de pozos excavados dentro del área de estudio que contaban con equipo de bombeo. Las condiciones de estos pozos es que son pozos excavados, sin tubería de revestimiento ni rejilla, son parcialmente 


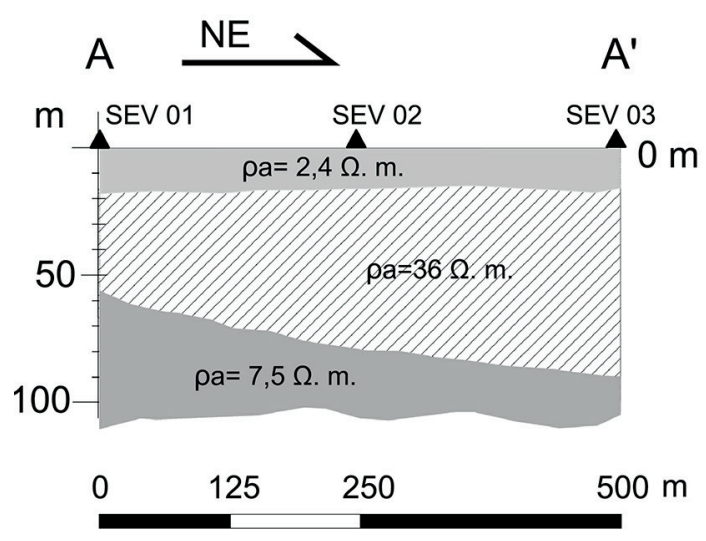

Fig. 4: Perfil geo eléctrico A-A'.

penetrantes en el acuífero, puesto que no exceden los $6 \mathrm{~m}$ de profundidad siendo el espesor de este acuífero de $20 \mathrm{~m}$ aproximadamente. Los pozos en los que se pudieron realizar las pruebas de bombeo son los únicos 3 pozos que cuentan con equipo de bombeo en la zona de estudio, los valores obtenidos en estos pozos se muestran en el cuadro 1 que se muestra a continuación:

Considerando que los datos obtenidos son puntuales, se interpreta que la transmisividad del acuífero superficial es de mediana producción en la parte alta de la cuenca y disminuye hacia la parte baja de la misma, lo cual es acorde al perfil litológico del área puesto que se observa una disminución en el espesor de la capa acuífera hacia el sur del área de estudio.

Esta zona ha sido poco explotada en cuanto al recurso hídrico subterráneo, de hecho el acuífero utilizado por la población para su abastecimiento es el acuífero somero. Los pozos excavados en la zona de estudio poseen profundidades menores a los $10 \mathrm{~m}$, no cuentan con revestimiento alguno, algunos poseen brocal hecho de ladrillo, la mayoría no tienen tapadera y están expuestos a la intemperie. Con la información de 74 pozos inventariados que se ubican en la figura 5 , se construyó la red de flujo. La figura 6, muestra una tendencia general del movimiento del agua subterránea del Noreste-Suroeste en el acuífero superficial. Lo cual indica una tendencia a seguir el drenaje natural del río Las Marías, tal y como también lo muestra el Mapa Hidrogeológico de El Salvador.
Cuadro 1

Resultados de pruebas de bombeo en pozos excavados

\begin{tabular}{cccccc}
\hline Pozo & Norte & Este & $\begin{array}{c}\text { NE } \\
(\mathrm{m})\end{array}$ & $\begin{array}{c}\mathrm{Q} \\
(\mathrm{l} / \mathrm{s})\end{array}$ & $\begin{array}{c}\mathrm{T} \\
\left(\mathrm{m}^{2} / \mathrm{d}\right)\end{array}$ \\
\hline PE-B21 & 276956 & 415060 & 2,84 & 1,95 & 106,3 \\
PE-B32 & 269578 & 413157 & 3,1 & 0,73 & 52,5 \\
PE-B20 & 267720 & 412926 & 3,33 & 0,46 & 15,3 \\
\hline
\end{tabular}

NE: nivel estático

\section{Rasgos hidrogeoquímicos}

Con el objetivo de realizar una clasificación hidrogeoquímica del agua subterránea del acuífero superficial, se realizó una campaña de muestreo de agua en octubre del año 2010, en total se recolectaron y analizaron 10 muestras de agua, todas provienen de pozos excavados como se observa en la figura 7 donde se presentan los puntos de muestreo. Posteriormente se realizaron análisis químicos de aniones y cationes mayoritarios, los análisis químicos se realizaron en el Laboratorio de Servicios Analíticos de la Fundación PROCAFE en El Salvador. Durante la recolección de las muestras se midieron en campo los parámetros de $\mathrm{pH}$, temperatura y conductividad eléctrica, utilizando un equipo multiparámetro facilitado por la Escuela Centroamericana de Geología de la Universidad de Costa Rica.

Acorde al diagrama de Piper mostrado en la figura 8 , se observa que las aguas subterráneas del área de estudio se pueden agrupar en dos tipos: bicarbonatadas cálcica - magnésica, lo cual denota que se trata de aguas jóvenes o recientes sin evolución química, provenientes de recarga local, este grupo incluye las muestras 2, 5, 6, 7, 8,9 y 10. El segundo grupo está compuesto por las muestras 1,3 y 4 , las cuales se clasifican como aguas cloruradas magnésicas, debido a que los cloruros son el anión dominante (más del $50 \%$ del total). Las diferencias en la distribución de los iones mayores en la zona se muestra en la figura 6 , donde se puede apreciar que las muestras 1, 3 y 4 no siguen el mismo patrón que las demás, lo cual se atribuye a contaminación puntual debido a la actividad antropogénica, es importante indicar que algunos de los pozos muestreados se ubican 


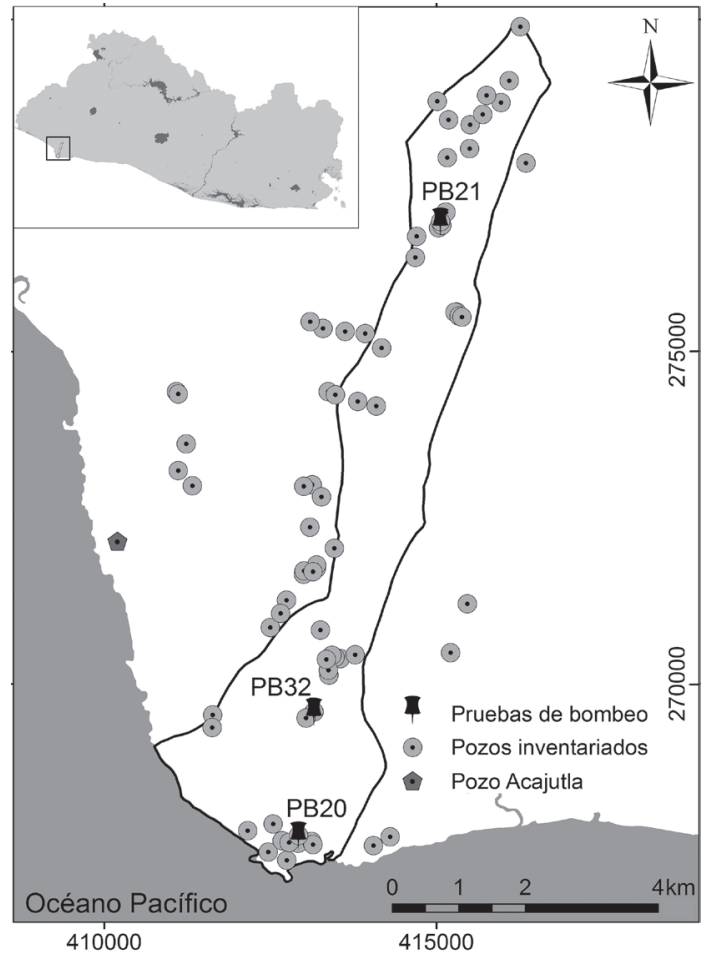

Fig. 5: Inventario de pozos y pruebas de bombeo.

en potreros por lo que podría haber contaminación puntual asociada al mal manejo de aguas residuales o bien a actividades agropecuarias.

Conforme a la relación analizada de magnesio $(\mathrm{Mg})$ y calcio $(\mathrm{Ca})$, se observa que los valores resultantes entre 0,25 y 0,61 de cada una de a las muestras corresponden a aguas continentales, dado que se obtuvieron valores dentro del rango de 0,25 a 1,5 .

$\mathrm{Al}$ evaluar la relación entre cloruro (Cl-) y bicarbonato $\left(\mathrm{HCO}^{3-}\right)$, en las muestras de agua subterránea se obtuvieron valores en el rango entre 0,06 a 0,76 considerando que valores indicadores de intrusión salina son iguales o superiores a 20, se concluye que los resultados obtenidos en esta investigación son muy lejanos a los que indicarían intrusión salina en el acuífero.

De acuerdo con los resultados obtenidos se tiene que los constituyentes mayoritarios del agua y la conductividad eléctrica se encuentran dentro de los rangos establecidos para aguas continentales, en adición la localización de los pozos que

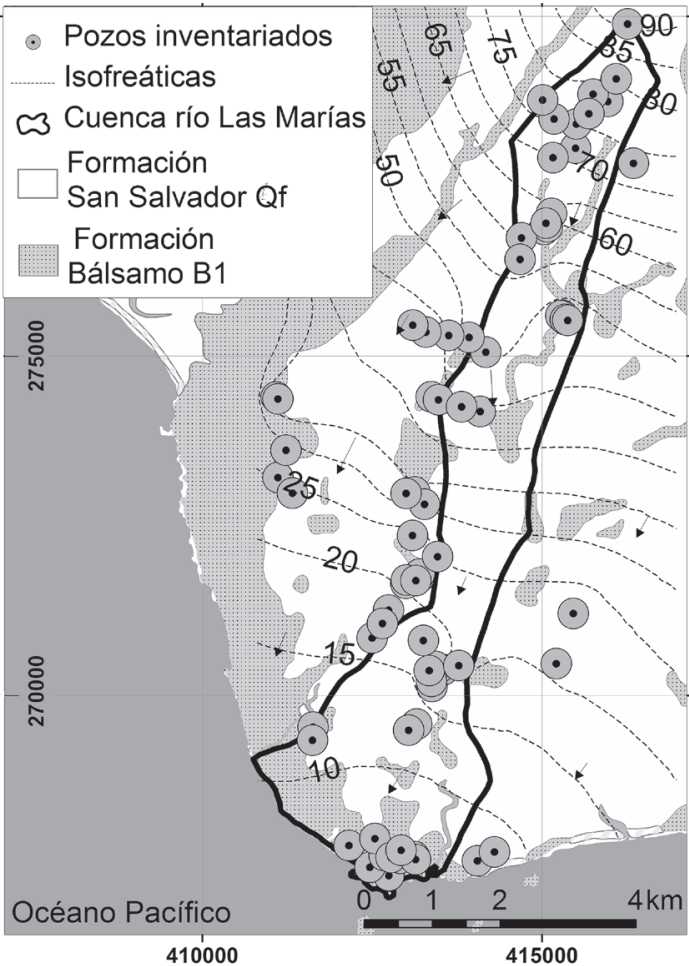

Fig. 6: Mapa hidrogeológico de la zona de estudio.

resultaron con tendencia clorurada no son los más cercanos a los límites de costa, por lo que se descarta que exista intrusión marina en el acuífero somero.

\section{Modelo hidrogeológico y evaluación de la intrusión salina}

Con base en la información recolectada se determinó que el acuífero somero presente en la zona de estudio, se ubica en los sedimentos aluviales (limos y arenas) intercalados con piroclastos (tobas retrabajadas, bloques de lava andesítica basáltica) que se observan aflorando en superficie, estos depósitos pueden alcanzar un espesor de hasta $20 \mathrm{~m}$ según la interpretación de los sondeos eléctricos verticales con resistividades que van de 6-15. Se determinó que este acuífero es libre, ya que no se reconoce ningún estrato confinante, además de los valores de transmisividad determinados de las pruebas en pozos excavados. 


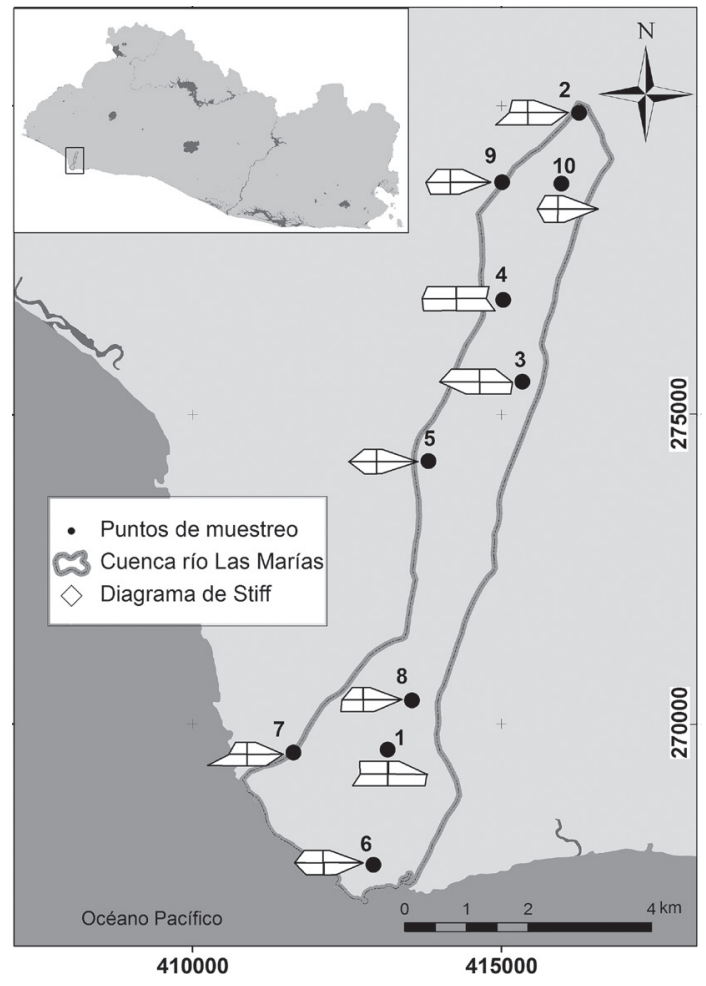

Fig. 7: Localización de puntos de muestreo y sus respectivos diagramas de Stiff.
La evidencia del acuífero profundo se obtuvo tanto de la interpretación de los sondeos eléctricos verticales como del nivel freático del pozo perforado en Acajutla, la profundidad del nivel de agua en el acuífero es de aproximadamente $30 \mathrm{~m}$ en esta zona, se considera que está acuífero es de carácter regional.

La información hidrogeoquímica y geofísica permitieron plantear el perfil del modelo conceptual, cuya línea de trazado se muestra en la figura 9 y posteriormente la conceptualización del perfil de la cuenca del río Las Marías se presenta en la figura 10.

\section{CONCLUSIONES}

Esta investigación permitió determinar la existencia de dos acuíferos, uno superficial de mediana a baja producción con caudales que van desde $\operatorname{los} 0,46$ a $1,95 \mathrm{l} / \mathrm{s}$, localizado a una profundidad de entre los $2 \mathrm{~m}$ a los $5 \mathrm{~m}$ con un espesor variable de hasta $20 \mathrm{~m}$, ubicado en los sedimentos aluviales (limos y, arenas) intercalados con piroclastos. Este acuífero es el más utilizado por la población de la zona como fuente de abastecimiento de agua por medio de pozos excavados manualmente en cada

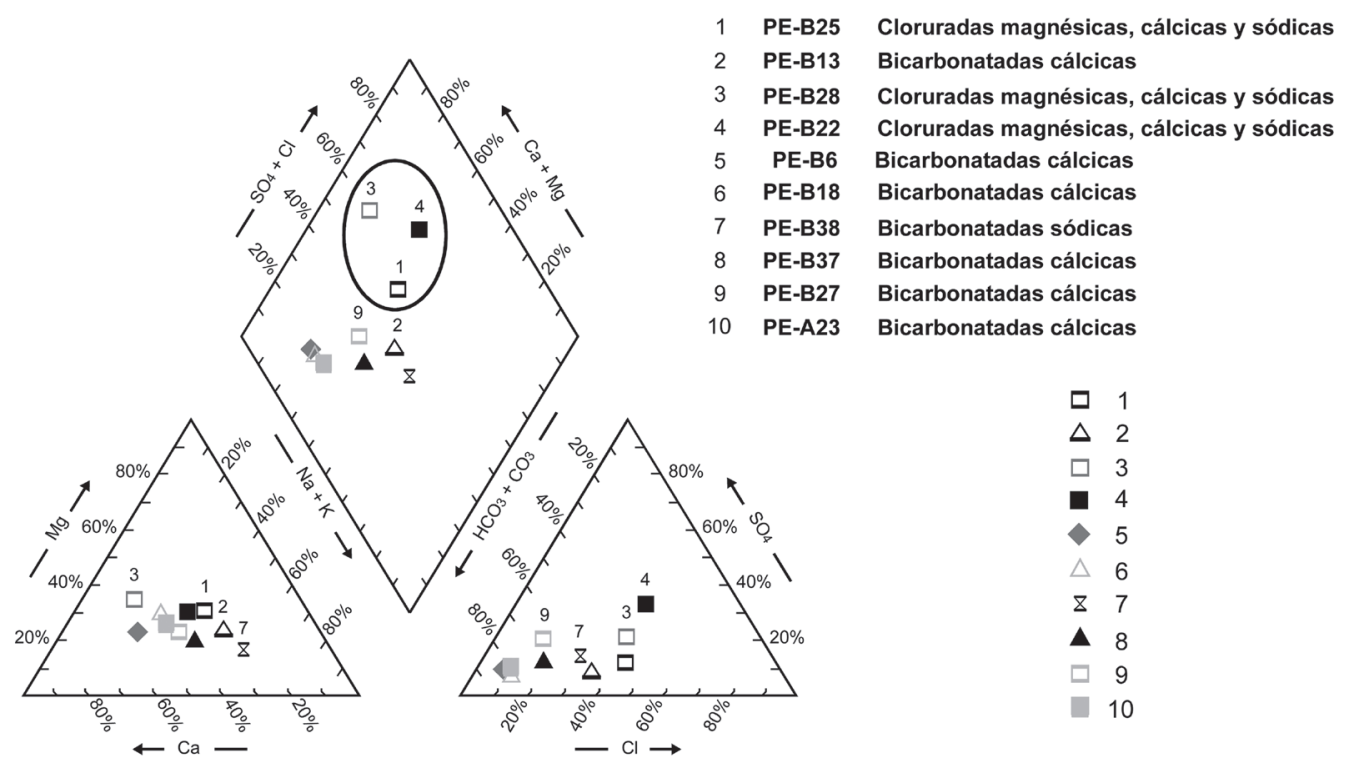

Fig. 8: Diagrama de Piper que muestra los resultados de los análisis químicos de las muestras de agua. 


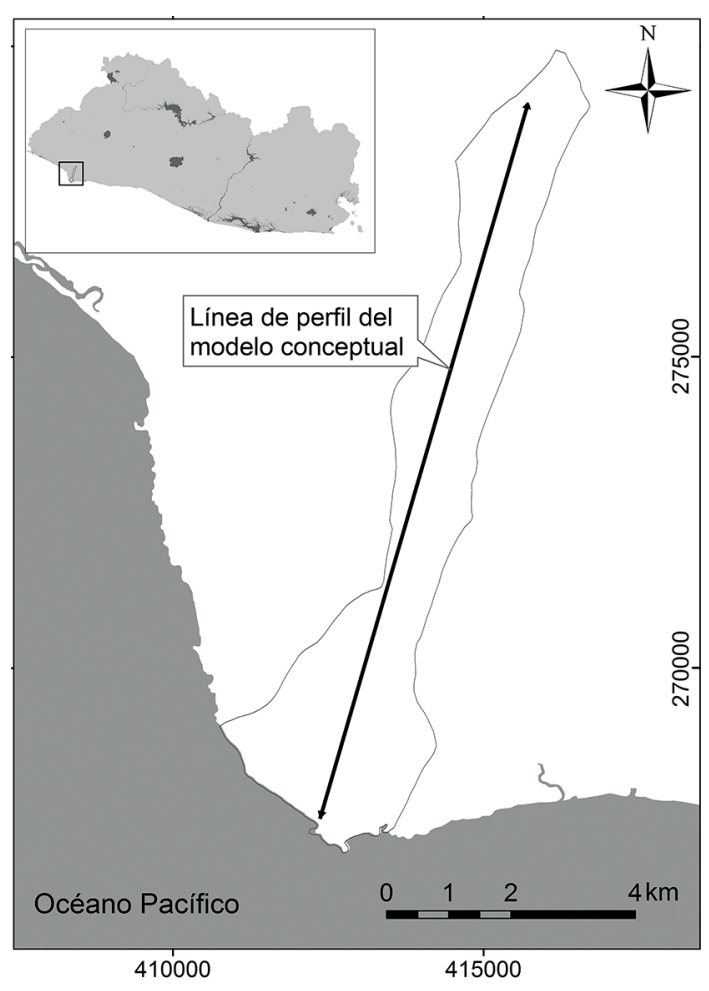

Fig. 9: Línea de perfil del modelo conceptual.

propiedad. Un segundo acuífero más profundo fue detectado con los sondeos eléctricos verticales y dentro del área de estudio se observó que existen pozos perforados privados que abastecen a las empresas hoteleras, fincas y residenciales privados del área, sin embargo los propietarios no accedieron a brindar información, por lo que solamente se dispone de la información litológica de un pozo en la zona de Acajutla.

Considerando que los datos obtenidos son puntuales producto de la poca disponibilidad de quipo para realizar las pruebas de bombeo, se interpreta que la transmisividad del acuífero superficial es de mediana producción en la parte alta de la cuenca y disminuye hacia la parte baja, lo cual es acorde al perfil litológico del área puesto que se observa una disminución en el espesor de la capa acuífera hacia el sur del área de estudio.

Los resultados de resistividad obtenidos de la prospección geofísica próxima a la costa, en donde se obtuvo una profundidad teórica de $100 \mathrm{~m}$, no reflejan que se haya interceptado la cuña de intrusión salina para el acuífero somero, lo que coincidió con los resultados hidrogeoquímicos, los cuales indican que los valores de los constituyentes mayoritarios del agua y la conductividad eléctrica se encuentran dentro de los rangos de aguas continentales. Por lo que se concluye que no hay intrusión salina al momento de la investigación.

Los valores altos de conductividad, cloruros y sólidos totales disueltos encontrados en tres de las muestras, que se ubican en potreros, indican contaminación del agua del acuífero somero, esto deberá analizarse con detalle en estudios futuros, para determinar las potenciales fuente de contaminación.

Conforme a los resultados del balance hídrico elaborado se obtuvo que de los $1739 \mathrm{~mm}$ de precipitación anual, el 70,6\% equivalente a $1217 \mathrm{~mm}$ corresponde a evapotranspiración real, el 13\% (226 $\mathrm{mm})$ pertenece a la retención por la vegetación y el restante $16,4 \%(276 \mathrm{~mm})$ representa la recarga acuífera de la zona. Como se puede notar no se tiene un valor de escurrimiento dado que la zona es muy plana y cuando el suelo alcanza la saturación la zona se encuentra anegada lo que mantiene aún las condiciones de humedal y su biodiversidad.

La red de flujo construida con la información recopilada muestra una tendencia general del movimiento del agua subterránea en el acuífero superficial del Noreste-Suroeste. Las líneas de flujo indican una tendencia a seguir el drenaje natural del río Las Marías, con un gradiente hidráulico del $0,37 \%$ cambiando hacia el suroeste como se corrobora en el Mapa Hidrogeológico de El Salvador.

Se recomienda que se construyan al menos tres pozos de observación en la zona, ubicados en la parte alta, media y baja de la cuenca, en donde además se cuente con un registro litológico continuo que brinde información de la geología de la zona en profundidad, esto brindará información clave acerca de la presencia del acuífero profundo.

\section{REFERENCIAS BIBLIOGRÁFICAS}

ANDA-COSUDE, 2008: Mapa Hidrogeológico de El Salvador.- Mapa escala 1:100 000, Water and Earth Science Associates Ltd., San Salvador. 


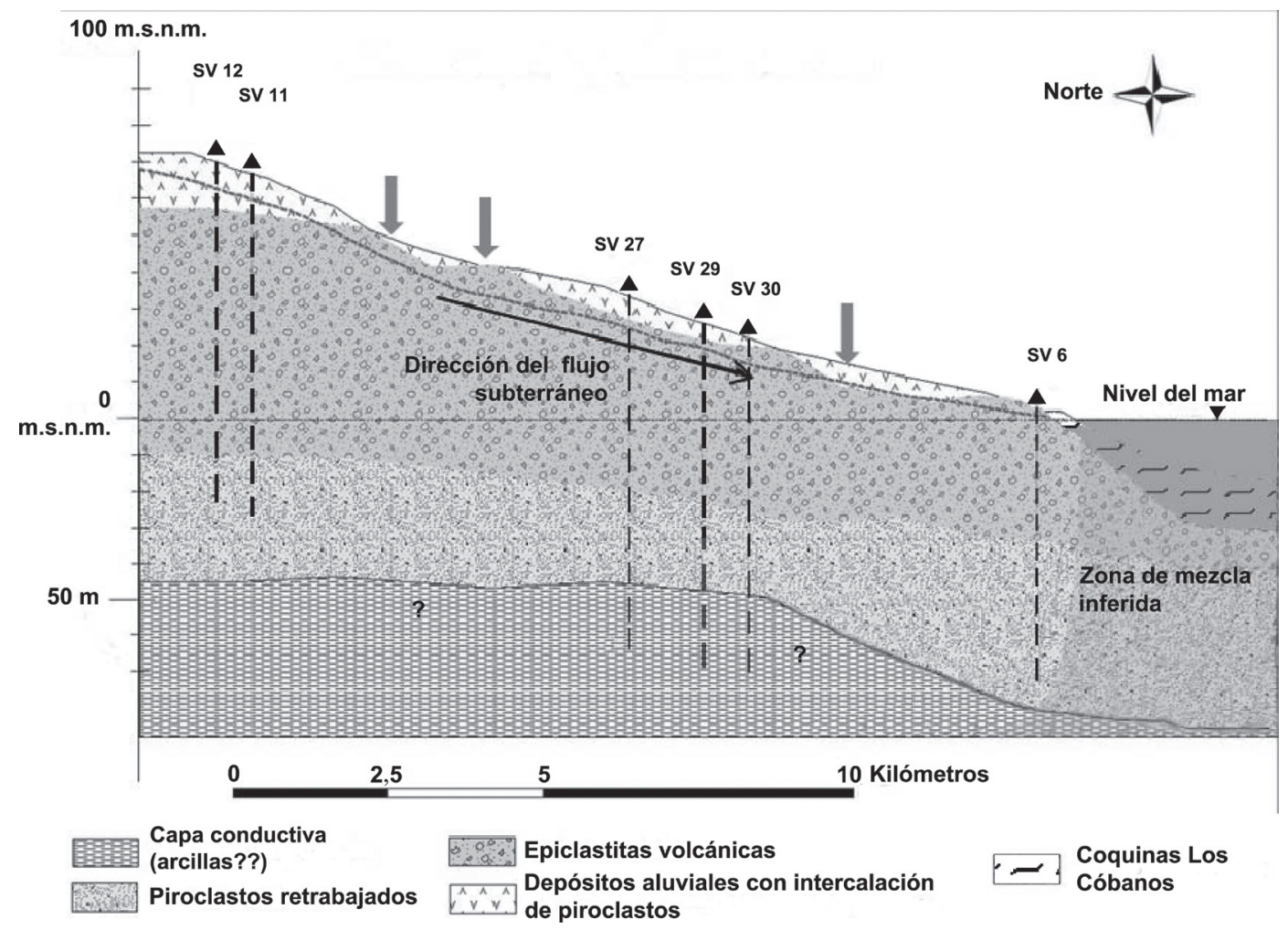

Fig. 10: Modelo conceptual de la cuenca Las Marías.

BAXTER, S., 1984: Léxico Estratigráfico de El Salvador.- 108 págs. Comisión Ejecutiva Hidroeléctrica del río Lempa (CEL), San Salvador.

DÜRR, F., 1960: Energía Geotérmica, Informe Geotérmico No.1.- 268 págs. Servicio Geológico Nacional. San Salvador, El Salvador. [Inf. interno].

ESTEVEZ, J., 2007: Estudio hidrogeológico del área planta de almacenamiento y distribución de combustibles derivados del petróleo Acajutla, Departamento de Sonsonate.- 42 Págs. Administración Nacional de Acueductos y Alcantarillados. San Salvador, El Salvador [Inf. interno].
KEAREY, P., BROOKS, M., \& Hill, I., 2002: An Introduction to Geophysical Exploration [3rd ed.].- 272 págs. Blackwell Science Ltd., Oxford.

GEOCEL, 1992: Estudio hidrogeológico para abastecimiento de agua a la central térmica Acajutla.- 67 págs. Comisión Ejecutiva Hidroeléctrica del Rio Lempa (CEL). Santa Ana, El Salvador [Inf. interno].

MACHUCA, W. E., 2003: Estudio hidrogeológico del área del cantón Punta Remedios - Playa Los Cóbanos, J/Acajulta, Departamento de Sonsonate.- 15 Págs. Administración Nacional de Acueductos y Alcantarillados. San Salvador, El Salvador [Inf. Interno]. 
MARN, 2007: Decreto ejecutivo No.22. Declaratoria del Complejo Los Cóbanos como primer área natural marino costera protegida de El Salvador.- Diario Oficial (SV). Set. 04: 43-143 (No.29 Tomo 378).

MISIÓN GEOLÓGICA ALEMANA EN EL SALVADOR, 1978: Mapa Geológico de la República de El Salvador.- Mapa escala 1:100 000. Bund. F. Geow. U. Rohs. Hannover.

PNUD, 1981: Plan Maestro de Desarrollo y Aprovechamiento de los Recursos
Hídricos. Documento Básico No.6. Recursos y Demandas Potenciales en la Región "D".- 44 págs. San Salvador, El Salvador [Inf. interno].

SCHOSINSKY, G., 2006: Cálculo de la recarga potencial de acuíferos mediante un balance hídrico de suelos.- Rev. Geol. Amér. Central 34 - 35: 13-30.

WIESEMAN, G., 1975. Remarks on the Geologic Structure of the Republic of El Salvador, Central America.- Mitt. Geol. Paläont. Inst. 44: 557-574. 
